\title{
IMPROVED BOUNDS FOR THE RIP OF SUBSAMPLED CIRCULANT MATRICES
}

\author{
MENG HUANG, YUXUAN PANG, AND ZHIQIANG XU
}

\begin{abstract}
In this paper, we study the restricted isometry property of partial random circulant matrices. For a bounded subgaussian generator with independent entries, we prove that the partial random circulant matrices satisfy $s$-order RIP with high probability if one chooses $m \gtrsim s \log ^{2}(s) \log (n)$ rows randomly where $n$ is the vector length. This improves the previously known bound $m \gtrsim s \log ^{2} s \log ^{2} n$.
\end{abstract}

\section{INTRODUCTION}

1.1. Compressed sensing. The aim of compressed sensing $[6,9,13,22]$ is to recover $s$ sparse signals $\mathbf{x} \in \mathbb{C}^{n}$ from the linear measurements $\mathbf{y}=\mathbf{A} \mathbf{x}$ and the compressed sensing matrix $\mathbf{A} \in \mathbb{C}^{m \times n}$ with $m<n$. Here, we say $\mathbf{x} \in \mathbb{C}^{n}$ is $s$-sparse if $\|\mathbf{x}\|_{0} \leq s$ where $\|\mathbf{x}\|_{0}$ denotes the number of nonzero entries of $\mathbf{x}$. A naive approach for reconstructing $\mathbf{x}$ is to solve the following $\ell_{0}$-minimization problem

$$
\left(P_{0}\right) \quad \min _{\mathbf{z}}\|\mathbf{z}\|_{0} \quad \text { subject to } \quad \mathbf{A z}=\mathbf{y} .
$$

However, the $\ell_{0}$-minimization problem is NP-hard [20] which is not tractable. A natural approach is to relax $\ell_{0}$-minimization to $\ell_{1}$-minimization $[6,8,9,13]$, i.e.,

$$
\left(P_{1}\right) \quad \min _{\mathbf{z}}\|\mathbf{z}\|_{1} \quad \text { subject to } \quad \mathbf{A z}=\mathbf{y} .
$$

The $\left(P_{1}\right)$ is a convex optimization problem which can be solved efficiently. To guarantee the reconstruction of the sparse signals $\mathbf{x}$ by $\left(P_{1}\right)$, it suffices to require the matrix $\mathbf{A}$ satisfying restricted isometry property (RIP). For a matrix $\mathbf{A} \in \mathbb{R}^{m \times n}$ and an integer $s \in[1, n)$, we say A satisfies $s$-order RIP with constant $\delta_{s} \in[0,1)$ if

$$
\left(1-\delta_{s}\right)\|\mathbf{x}\|_{2}^{2} \leq\|\mathbf{A} \mathbf{x}\|_{2}^{2} \leq\left(1+\delta_{s}\right)\|\mathbf{x}\|_{2}^{2}
$$

holds for all $s$-sparse signals $\mathbf{x}$. It has been proved that if the matrix $\mathbf{A}$ satisfies $t \cdot s$-order RIP with $\delta_{t s}<\sqrt{1-\frac{1}{t}}$ for $t>4 / 3$, then every $s$-sparse vector $\mathbf{x}$ can be reconstructed

Zhiqiang Xu was supported by NSFC grant (91630203, 11331012, 11688101) and by National Basic Research Program of China (973 Program 2015CB856000). 
by solving $\left(P_{1}\right)[5,7]$. Moreover, RIP is also employed to study the performance of greedy algorithms for the recovery of sparse signals, such as OMP [26], OMMP [25], CoSaMP $[11,21]$, iterative hard thresholding [3] and hard thresholding pursuit [10]. Hence, one is interested in constructing RIP matrices $\mathbf{A} \in \mathbb{C}^{m \times n}$ with $m$ being as small as possible. A popular method for constructing RIP matrices is to use random matrices. For example, Gaussian random matrix $\frac{1}{\sqrt{m}} \mathbf{A} \in \mathbb{R}^{m \times n}$ satisfies $s$-order RIP with high probability provided that $m \geq C s \log (n / s)$, where the entries of $\mathbf{A}$ are independent standard normal random variables. From the Gelfand widths, the lower bound $C s \log (n / s)$ is optimal up to a constant [12]. Others random matrices which can achieve this bound include Bernoulli matrices and subgaussian matrices $[18,22]$. In practical applications, one prefers structure random matrices since they can make the recovery algorithms more efficient. An important example of structure random matrices is partial Fourier matrices whose rows are selected randomly from the discrete Fourier matrix. It has been showed that the partial Fourier matrices satisfy $s$-order RIP with high probability provided that $m \geq C s \log ^{2} s \log n,($ see $[4,15,22,24])$.

The aim of this paper is to study the RIP of the partial random circulant matrices. Compared to Bernoulli or Gaussian matrices, the partial random circulant matrices have the advantage that they reduce the generation of only $n$ independent random variables instead of $n^{2}$. More importantly, they admit fast matrix-vector multiplication and arise naturally in certain applications such as in radar, aperture imaging [14,23] as well as MR imaging [17]. Hence, they attract much attention.

1.2. Related work. Assume that $\xi=\left(\xi_{1}, \ldots, \xi_{n}\right) \in \mathbb{C}^{n}$. We define the circulant matrix generated by $\xi$ as

$$
\mathbf{A}_{\xi}:=\left(\begin{array}{cccc}
\xi_{1} & \xi_{n} & \cdots & \xi_{2} \\
\xi_{2} & \xi_{1} & \cdots & \xi_{3} \\
\vdots & \vdots & \ddots & \vdots \\
\xi_{n} & \xi_{n-1} & \cdots & \xi_{1}
\end{array}\right) \in \mathbb{C}^{n \times n}
$$

For a multiset $\Omega$ in $\{1, \ldots, n\}$ with cardinality $m$, let $P_{\Omega}: \mathbb{C}^{n} \rightarrow \mathbb{C}^{m}$ denote the projection operator that restricts a vector $\mathbf{x} \in \mathbb{C}^{n}$ to its entries in $\Omega$. Then the corresponding partial circulant matrix generated by $\xi$ is defined as

$$
\Phi_{\xi}=\frac{1}{\sqrt{m}} P_{\Omega} \mathbf{A}_{\xi} \in \mathbb{C}^{m \times n} .
$$


We next introduce another structured random matrices. For $\xi=\left(\xi_{1}, \ldots, \xi_{2 n-1}\right) \in \mathbb{C}^{2 n-1}$, the Toeplitz matrix $T_{\xi}$ is defined as

$$
T_{\xi}=\left(\begin{array}{cccc}
\xi_{n} & \xi_{n-1} & \cdots & \xi_{1} \\
\xi_{n+1} & \xi_{n} & \cdots & \xi_{2} \\
\vdots & \vdots & \ddots & \vdots \\
\xi_{2 n-1} & \xi_{2 n-2} & \cdots & \xi_{n}
\end{array}\right)
$$

and the Hankel matrix is defined as $H_{\xi}=T_{\xi} J_{n}$ where $J_{n}=\left[\mathbf{e}_{n}, \ldots, \mathbf{e}_{1}\right]$ and $\mathbf{e}_{j}, j=1, \ldots, n$ are the standard orthogonal vectors. For a multiset $\Omega$ of $\{1, \ldots, n\}$ with cardinality $m$, the corresponding partial Toeplitz matrix

$$
\Psi_{\xi}=\frac{1}{\sqrt{m}} P_{\Omega} T_{\xi} \in \mathbb{C}^{m \times n}
$$

Similarly, we can define the partial Hankel matrix. A simple observation is that $\frac{1}{\sqrt{m}} P_{\Omega} T_{\xi}$ satisfies $s$-order RIP with the constant $\delta_{s}$ if and only if $\frac{1}{\sqrt{m}} P_{\Omega} H_{\xi}$ satisfies the same property. As we will see later, a Toeplitz matrix can be embedded in a circulant matrix of twice the dimension. Hence, many RIP results for partial random circulant matrices can be extended to the partial random Toeplitz matrices as well as partial random Hankel matrices.

In [23], under the setup in which both $\Omega$ as well as the generating vector $\xi$ are chosen at random, Romberg proves that $m \gtrsim s \log ^{6} n$ measurements are sufficient to guarantee $\Phi_{\xi}=$ $\frac{1}{\sqrt{m}} P_{\Omega} \mathbf{A}_{\xi} \in \mathbb{C}^{m \times n}$ satisfying RIP. For an arbitrary fixed selection of $\Omega \subset\{1, \ldots, n\}$, the first theoretical results are established in [2] with showing that $m \times n$ partial random circulant matrices satisfy $s$-order RIP with high probability provided $m \gtrsim s^{3} \log n$. This is then improved by Bajwa et al. $[1,14]$ to $m \gtrsim s^{2} \log n$ for general matrices whose entries were drawn from bounded or Gaussian distribution. Later, Rauhut, Romberg and Tropp prove that $m \gtrsim s^{3 / 2} \log ^{3 / 2} n$ measurements are enough. And the result is then improved by Krahmer, Mendelson and Rauhut [16] to $O\left(s \log ^{2} s \log ^{2} n\right)$. Recently, Mendelson, Rauhut and Ward [19] prove that $O\left(s \log ^{2} s \log (\log s) \log n\right)$ measurements for partial random circulant matrix with random sampling set $\Omega$ and Gaussian random generator are sufficient to recover all $s$-sparse vectors with high probability via $\ell_{1}$-minimization, but it does not establish any type of RIP.

1.3. Main results. In this paper, we study the RIP of the partial random circulant matrices with bounded or Gaussian entries, where the subsampling locations $\Omega$ are selected at random from $\{1, \ldots, n\}$. We show that, if $m \gtrsim s \log ^{2} s \log n$ then $\Phi_{\xi}=\frac{1}{\sqrt{m}} P_{\Omega} \mathbf{A}_{\xi}$ satisfies 
$s$-order RIP with high probability where $\xi$ is a bounded random vector. This improves upon the best previously known bound $O\left(s \log ^{2} s \log ^{2} n\right)$ [16]. Some suitable bounded random vectors $\xi$ include uniform distribution $\xi_{i} \sim \mathrm{U}(-\sqrt{3}, \sqrt{3})$ and Rademacher vector with $\mathbb{P}\left(\xi_{i}= \pm 1\right)=1 / 2$.

Theorem 1.1. Let $\xi=\left(\xi_{1}, \ldots, \xi_{n}\right) \in \mathbb{C}^{n}$ be a random vector whose entries are i.i.d. realizations of bounded zero-mean random variables satisfying $\mathbb{E} \xi_{j}^{2}=1$ and $|\xi|_{j} \leq c$ for some $c \geq 1$. Suppose that $\delta>0$ is a sufficient small constant and $m \gtrsim \log ^{2}(1 / \delta) \cdot \delta^{-2} s \log ^{2}(s / \delta) \log n$. Let $\Omega$ be a multiset of $m$ uniform and independent random elements of $\{1, \ldots, n\}$ and $\Phi_{\xi}=\frac{1}{\sqrt{m}} P_{\Omega} \mathbf{A}_{\xi} \in \mathbb{C}^{m \times n}$ be a partial random circulant matrix generated by $\xi$ and $\Omega$. If $s \lesssim n /\left(\log ^{4} n\right)$, then the matrix $\Phi_{\xi}$ satisfies the restricted isometry property with order $s$ and constant $\delta_{s} \leq \delta$ with probability at least $1-2^{-C \log n \log (s / \delta)}-n^{-\log n \log ^{2} s}$.

In the practical applications, one usually requires that $s<<n$. Hence, the assumption of $s \lesssim n / \log ^{4} n$ in Theorem 1.1 is enough for the practical applications. The idea for proving Theorem 1.1 is to use result from [15] (see Theorem 2.4 in Section 2), which shows the connection between $\frac{1}{n}\|M \mathbf{x}\|_{2}^{2}$ and $\frac{1}{m}\left\|P_{\Omega} M \mathbf{x}\right\|_{2}^{2}$ for any matrix $M \in \mathbb{C}^{n \times n}$ (see (2.1) for detail). In [15], Haviv and Regev consider the case where $M$ is a DFT matrix for which $\frac{1}{n}\|M \mathbf{x}\|_{2}^{2}=\|\mathbf{x}\|_{2}^{2}$. A particular case of Theorem 2.2 from [16] shows that the circulant matrix $\mathbf{A}_{\xi}$ satisfies $\frac{1}{n}\left\|\mathbf{A}_{\xi} \mathbf{x}\right\|^{2} \approx\|\mathbf{x}\|^{2}$ provided $s \lesssim n / \log ^{4} n$ (see Corollary 2.3). Combining them, we can arrive at Theorem 1.1. The proof just combines the known results, but Theorem 1.1 definitely goes far beyond the current state-of-the-art.

Remark 1.2. We consider the case with removing the assumption of $\xi_{j}$ being a bounded random variable. Assume that $\xi \in \mathbb{C}^{n}$ is a random vector with independent, mean 0 and variance 1 , L-subgaussian entries. We can obtain that $\mathbb{P}\left(\left|\xi_{j}\right|>L \sqrt{2 \log n}\right) \leq 2 / n^{2}$ for any $\xi_{j}$. Then

$$
\mathbb{P}\left(\max _{j}\left|\xi_{j}\right| \leq L \sqrt{2 \log n}\right) \geq 1-2 / n,
$$

which implies that $\left|\xi_{j}\right| \leq L \sqrt{2 \log n}$ for all $j=1, \ldots, n$ with probability at least 1 $2 / n$. Since the bound $L \sqrt{2 \log n}$ is not a constant, it leads to slightly larger samples $m \gtrsim$ $s \log ^{2}(s \log n) \log ^{2} n$ for the partial random circulant matrix $\Phi_{\xi}$ to satisfy s-order RIP. Nevertheless, the result can match the best bound $m \gtrsim s \log ^{2}(s) \log ^{2}(n)$ which is obtained in [16] provided $s \geq \log n$. 
Remark 1.3. Note that a Toeplitz matrix can be embedded in a circulant matrix of twice the dimension. Then we can show that Toeplitz matrix satisfies $\frac{1}{n}\left\|T_{\xi} \mathbf{x}\right\|^{2} \approx\|\mathbf{x}\|^{2}$ provided $s \lesssim n / \log ^{4} n$. Hence, our result can be extended to the partial random Toeplitz matrices.

\section{Preliminaries}

We first introduce the definition of $L$-subgaussian random vectors which include the Rademacher vectors as well as the standard Gaussian vectors as special cases. To state conveniently, we use $\mathcal{S}^{n-1}$ to denote the unit sphere in $\mathbb{C}^{n}$.

Definition 2.1 ( $L$-subgaussian). A mean 0 random vector $X \in \mathbb{C}^{n}$ is called isotropic if for every $\theta \in \mathcal{S}^{n-1}, \mathbb{E}|\langle X, \theta\rangle|=1$. A random vector $X$ is called $L$-subgaussian if it is isotropic and $\mathbb{P}(|\langle X, \theta\rangle| \geq t) \leq 2 \exp \left(-t^{2} / 2 L^{2}\right)$ for every $\theta \in \mathcal{S}^{n-1}$ and any $t \geq 0$.

We introduce some known results which are useful in our analysis.

Theorem 2.2. ([16, Theorem 4.1]) For any fixed subset $\Omega \subset\{1, \ldots, n\}$ and a random vector $\xi \in \mathbb{C}^{n}$ with independent, mean 0 and variance 1 , L-subgaussian entries. Let $\Phi_{\xi}=$ $\frac{1}{\sqrt{m}} P_{\Omega} \mathbf{A}_{\xi} \in \mathbb{R}^{m \times n}$ be a partial random circulant matrix generated by $\xi$ and $\Omega$. If

$$
m \geq c \delta^{-2} s \log ^{2} s \log ^{2} n,
$$

then with probability at least $1-n^{-\log n \log ^{2} s}$, the matrix $\Phi_{\xi}$ satisfies the restricted isometry property with constant $\delta_{s} \leq \delta$. The constant $c>0$ is universal.

If we take $\Omega=\{1, \ldots, n\}$, then we have the following corollary:

Corollary 2.3. Suppose that $\xi \in \mathbb{C}^{n}$ is a random vector with independent, mean 0 and variance 1, L-subgaussian entries. Suppose that $s \lesssim n / \log ^{4} n$. Then

$$
(1-\delta)\|\mathbf{x}\|^{2} \leq \frac{1}{n}\left\|\mathbf{A}_{\xi} \mathbf{x}\right\|^{2} \leq(1+\delta)\|\mathbf{x}\|^{2} \quad \text { for all } s \text {-sparse } \mathbf{x} \in \mathbb{C}^{n}
$$

holds with probability at least $1-n^{-\log n \log ^{2} s}$.

The above corollary shows that we can obtain $(1-\delta)\|\mathbf{x}\|^{2} \leq \frac{1}{n}\left\|\mathbf{A}_{\xi} \mathbf{x}\right\|^{2} \leq(1+\delta)\|\mathbf{x}\|^{2}$ for all $s$-sparse vector $\mathbf{x}$ provided $s \lesssim n / \log ^{4} n$.

We next introduce the main result in [15] which also plays an important role in our analysis. 
Theorem 2.4. ([15, Theorem 4.1]) For a sufficiently large $n$, a matrix $M \in \mathbb{C}^{n \times n}$, and sufficiently small $\varepsilon, \eta>0$, the following holds. For some $m=O\left(\log ^{2}(1 / \varepsilon) \cdot \varepsilon^{-1} \eta^{-1} \log n \cdot \log ^{2}(1 / \eta)\right)$, let $\Omega$ be a multiset of $m$ uniform and independent random elements of $\{1, \ldots, n\}$. Then, with probability $1-2^{-\Omega(\log n \cdot \log (1 / \eta))}$, it holds that for every $\mathbf{x} \in \mathbb{C}^{n}$,

(2.1) $\frac{1}{n}(1-\varepsilon)\|M \mathbf{x}\|_{2}^{2}-\eta\|\mathbf{x}\|_{1}^{2}\|M\|_{\infty}^{2} \leq \frac{1}{m} \sum_{j \in \Omega}\left|(M \mathbf{x})_{j}\right|^{2} \leq \frac{1}{n}(1+\varepsilon)\|M \mathbf{x}\|_{2}^{2}+\eta\|\mathbf{x}\|_{1}^{2}\|M\|_{\infty}^{2}$, where $\|M\|_{\infty}:=\max _{i, j}\left|M_{i, j}\right|$.

\section{Proofs of Theorem 1.1}

Before giving the proof of Theorem 1.1, we next introduce a proposition which shows that $\frac{1}{n}\left\|\mathbf{A}_{\xi} \mathbf{x}\right\|^{2} \approx\|\mathbf{x}\|^{2}$ does not hold for all $\mathbf{x} \in \mathbb{C}^{n}$ with high probability. Hence, to guarantee $\frac{1}{n}\left\|\mathbf{A}_{\xi} \mathbf{x}\right\|^{2} \approx\|\mathbf{x}\|^{2}$ we need to require $\mathbf{x}$ lies in some subset in $\mathbb{C}^{n}$. For example, in Theorem 1.1, we require $s=\|\mathbf{x}\|_{0} \lesssim n / \log ^{4} n$. This also shows the essential difference between $\mathbf{A}_{\xi}$ and Fourier matrices.

To state conveniently, we say a vector is a Gaussian random vector if the entries are i.i.d. standard Gaussian random variables.

Proposition 3.1. Let $\xi=\left(\xi_{1}, \ldots, \xi_{n}\right) \in \mathbb{R}^{n}$ be a Rademacher vector or Gaussian random vector and $\mathbf{A}_{\xi} \in \mathbb{R}^{n \times n}$ be the random circulant matrix generated by $\xi$. Then for any fixed $\epsilon>0$, there exists a vector $\mathbf{x} \in \mathbb{R}^{n}$ and a positive constant $p_{0}$ so that

$$
\mathbb{P}\left(\frac{1}{n}\left\|\mathbf{A}_{\xi} \mathbf{x}\right\|_{2}^{2}<\epsilon\right) \geq p_{0} \text { when } n \text { is large enough. }
$$

Proof. Setting $\mathbf{x}=\frac{1}{\sqrt{n}}(1, \ldots, 1)^{\top}$, we have

$$
\frac{1}{n}\left\|\mathbf{A}_{\xi} \mathbf{x}\right\|_{2}^{2}=\left(\frac{\xi_{1}+\cdots+\xi_{n}}{\sqrt{n}}\right)^{2}:=\zeta_{n}^{2} .
$$

For Gaussian random vector $\xi$, a simple observation is that $\zeta_{n} \sim \mathcal{N}(0,1)$. It implies that

$$
\mathbb{P}\left(\frac{1}{n}\left\|\mathbf{A}_{\xi} \mathbf{x}\right\|_{2}^{2}<\epsilon\right)=\mathbb{P}\left(\left|\zeta_{n}\right|<\sqrt{\epsilon}\right)=2(1-\Phi(\sqrt{\epsilon})):=p_{0},
$$

where $\Phi(x)$ is the cumulative distribution function of standard Gaussian random variable. For Rademacher vector $\xi$, note that $\left\{\xi_{i}\right\}$ is a i.i.d. random variable sequence with $\mathbb{E}\left(\xi_{i}\right)=0$ and $\mathbb{E}\left(\xi_{i}^{2}\right)=1$. Recall that

$$
\mathbb{P}\left(\frac{1}{n}\left\|\mathbf{A}_{\xi} \mathbf{x}\right\|_{2}^{2}<\epsilon\right)=\mathbb{P}\left(\left|\zeta_{n}\right|<\sqrt{\epsilon}\right) .
$$


Then from Central Limit Theorem, we obtain that $\mathbb{P}\left(\left|\zeta_{n}\right|<\sqrt{\epsilon}\right)$ tends to $2(1-\Phi(\sqrt{\epsilon}))$ with $n$ tending to infinity. Hence, we arrive at conclusion.

We next give the proof of Theorem 1.1.

Proof of Theorem 1.1. Through out this proof, we assume that $\|\mathbf{x}\|_{0} \leq s$. Recall that $\Phi_{\xi}=\frac{1}{\sqrt{m}} P_{\Omega} \mathbf{A}_{\xi}$. From Corollary 2.3, we obtain that

$$
(1-\delta / 4)\|\mathbf{x}\|_{2}^{2} \leq \frac{1}{n}\left\|\mathbf{A}_{\xi} \mathbf{x}\right\|_{2}^{2} \leq(1+\delta / 4)\|\mathbf{x}\|_{2}^{2}, \quad \text { for all } s \text {-sparse vectors } \mathbf{x}
$$

holds with probability at least $1-n^{-\log n \log ^{2} s}$ provided $s \lesssim \delta^{2} n /\left(\log ^{2} s \log ^{2} n\right)$. CauchySchwarz inequality implies that $\|\mathbf{x}\|_{1} \leq \sqrt{s}\|\mathbf{x}\|_{2}$. Observe that

$$
\left\|\Phi_{\xi} \mathbf{x}\right\|_{2}^{2}=\frac{1}{m} \sum_{j \in \Omega}\left|\left(\mathbf{A}_{\xi} \mathbf{x}\right)_{j}\right|^{2} .
$$

For the given multiset $\Omega$, choosing $\eta \geq \delta /\left(4 c^{2} s\right)$ in Theorem 2.4 for a fixed circulant matrix $\mathbf{A}_{\xi}$ with $\left\|\mathbf{A}_{\xi}\right\|_{\infty} \leq c$, then with probability at least $1-2^{-C \log n \log (s / \delta)}$ we have

$$
\frac{1}{n}(1-\delta / 4)\left\|\mathbf{A}_{\xi} \mathbf{x}\right\|_{2}^{2}-\delta / 4\|\mathbf{x}\|_{2}^{2} \leq\left\|\Phi_{\xi} \mathbf{x}\right\|_{2}^{2} \leq \frac{1}{n}(1+\delta / 4)\left\|\mathbf{A}_{\xi} \mathbf{x}\right\|_{2}^{2}+\delta / 4\|\mathbf{x}\|_{2}^{2}
$$

provided $m=O\left(\log ^{2}(1 / \delta) \cdot \delta^{-2} s \log ^{2}(s / \delta) \log n\right)$.

Combining (3.1) and (3.2), we obtain that

$$
(1-\delta)\|\mathbf{x}\|_{2}^{2} \leq\left\|\Phi_{\xi} \mathbf{x}\right\|_{2}^{2} \leq(1+\delta)\|\mathbf{x}\|_{2}^{2}
$$

holds with probability at least $1-2^{-C \log n \log (s / \delta)}-n^{-\log n \log ^{2} s}$, which arrives at the conclusion.

\section{REFERENCES}

[1] Waheed U Bajwa, Jarvis Haupt, Gil Raz, and Robert Nowak. Compressed channel sensing. In Information Sciences and Systems, 2008. CISS 2008. 42nd Annual Conference on, pages 5-10. IEEE, 2008.

[2] Waheed U Bajwa, Jarvis D Haupt, Gil M Raz, Stephen J Wright, and Robert D Nowak. Toeplitzstructured compressed sensing matrices. In Statistical Signal Processing, 200\%. SSP'07. IEEE/SP 14th Workshop on, pages 294-298. IEEE, 2007.

[3] Thomas Blumensath and Mike E Davies. Iterative hard thresholding for compressed sensing. Applied and computational harmonic analysis, 27(3):265-274, 2009.

[4] Jean Bourgain. An improved estimate in the restricted isometry problem. In Geometric Aspects of Functional Analysis, pages 65-70. Springer, 2014.

[5] T. Cai, A. Zhang. Sparse Representation of a Polytope and Recovery of Sparse Signals and Low-rank Matrices, IEEE Transactions on Information Theory 60(1):122-132, 2014. 
[6] Emmanuel J Candès, Justin Romberg, and Terence Tao. Robust uncertainty principles: Exact signal reconstruction from highly incomplete frequency information. IEEE Transactions on information theory, 52(2):489-509, 2006.

[7] E. J. Candès, J. Romberg, and T. Tao. Stable signal recovery from incomplete and inaccurate measurements. Comm. Pure Appl. Math., 59(8):1207-1223, 2006.

[8] Scott Shaobing Chen, David L Donoho, and Michael A Saunders. Atomic decomposition by basis pursuit. SIAM review, 43(1):129-159, 2001.

[9] David L Donoho. Compressed sensing. IEEE Transactions on information theory, 52(4):1289-1306, 2006.

[10] Simon Foucart. Hard thresholding pursuit: an algorithm for compressive sensing. SIAM Journal on Numerical Analysis, 49(6):2543-2563, 2011.

[11] Simon Foucart. Sparse recovery algorithms: sufficient conditions in terms of restricted isometry constants. In Approximation Theory XIII: San Antonio 2010, pages 65-77. Springer, 2012.

[12] Simon Foucart, Alain Pajor, Holger Rauhut, and Tino Ullich. The gelfand widths of $\ell_{p}$-balls for $0<$ $p \leq 1$. Journal of Complexity, 26(6):629-640, 2010.

[13] Simon Foucart and Holger Rauhut. A mathematical introduction to compressive sensing, volume 1. Birkhäuser Basel, 2013.

[14] Jarvis Haupt, Waheed U Bajwa, Gil Raz, and Robert Nowak. Toeplitz compressed sensing matrices with applications to sparse channel estimation. IEEE transactions on information theory, 56(11):5862-5875, 2010.

[15] Ishay Haviv and Oded Regev. The restricted isometry property of subsampled fourier matrices. In Geometric Aspects of Functional Analysis, pages 163-179. Springer, 2017.

[16] Felix Krahmer, Shahar Mendelson, and Holger Rauhut. Suprema of chaos processes and the restricted isometry property. Communications on Pure and Applied Mathematics, 67(11):1877-1904, 2014.

[17] Dong Liang, Guangwu Xu, Haifeng Wang, Kevin F King, Dan Xu, and Leslie Ying. Toeplitz random encoding $\mathrm{mr}$ imaging using compressed sensing. In Biomedical Imaging: From Nano to Macro, 2009. ISBI'09. IEEE International Symposium on, pages 270-273. IEEE, 2009.

[18] Shahar Mendelson, Alain Pajor, and Nicole Tomczak-Jaegermann. Uniform uncertainty principle for bernoulli and subgaussian ensembles. Constructive Approximation, 28(3):277-289, 2008.

[19] Shahar Mendelson, Holger Rauhut, and Rachel Ward. Improved bounds for sparse recovery from subsampled random convolutions. Annals of Applied Probability, accepted, 2016.

[20] Balas Kausik Natarajan. Sparse approximate solutions to linear systems. SIAM journal on computing, 24(2):227-234, 1995.

[21] Deanna Needell and Joel A Tropp. CoSaMP: Iterative signal recovery from incomplete and inaccurate samples. Applied and computational harmonic analysis, 26(3):301-321, 2009.

[22] Holger Rauhut. Compressive sensing and structured random matrices. Theoretical foundations and numerical methods for sparse recovery, 9:1-92, 2010.

[23] Justin Romberg. Compressive sensing by random convolution. SIAM Journal on Imaging Sciences, 2(4):1098-1128, 2009.

[24] Mark Rudelson and Roman Vershynin. On sparse reconstruction from Fourier and Gaussian measurements. Communications on Pure and Applied Mathematics, 61(8):1025-1045, 2008.

[25] Zhiqiang $\mathrm{Xu}$. The performance of orthogonal multi-matching pursuit under RIP, J. Comp. Math 33(2015), 495-516.

[26] Tong Zhang. Sparse recovery with orthogonal matching pursuit under rip. IEEE Transactions on Information Theory, 57(9):6215-6221, 2011. 
LSEC, Inst. Comp. Math., Academy of Mathematics and System Science, Chinese Academy of Sciences, Beijing, 100091, China

E-mail address: hm@lsec.cc.ac.cn

LSEC, Inst. Comp. Math., Academy of Mathematics and System Science, Chinese Academy of Sciences, Beijing, 100091, China

E-mail address: pangyuxuan@lsec.cc.ac.cn

LSEC, Inst. Comp. Math., Academy of Mathematics and System Science, Chinese Academy of Sciences, Beijing, 100091, China

School of Mathematical Sciences, University of Chinese Academy of Sciences, Beijing 100049, CHINA

E-mail address: xuzq@isec.cc.ac.cn 